\title{
CD19 is a major B cell receptor-independent activator of MYC-driven B-lymphomagenesis
}

\author{
Elaine Y. Chung, ${ }^{1}$ James N. Psathas, ${ }^{1}$ Duonan $\mathrm{Yu},{ }^{2}$ Yimei Li, ${ }^{3}$ \\ Mitchell J. Weiss, ${ }^{2,4}$ and Andrei Thomas-Tikhonenko ${ }^{1,4}$
}

\begin{abstract}
1Division of Cancer Pathobiology, Department of Pathology and Laboratory Medicine, 2Division of Hematology, and ${ }^{3}$ Division of Oncology, Department of Pediatrics, The Children's Hospital of Philadelphia, Philadelphia, Pennsylvania, USA ${ }^{4}$ Perelman School of Medicine at the University of Pennsylvania, Philadelphia, Pennsylvania, USA.
\end{abstract}

\begin{abstract}
PAX5, a B cell-specific transcription factor, is overexpressed through chromosomal translocations in a subset of $B$ cell lymphomas. Previously, we had shown that activation of immunoreceptor tyrosine-based activation motif (ITAM) proteins and B cell receptor (BCR) signaling by PAX5 contributes to B-lymphomagenesis. However, the effect of PAX5 on other oncogenic transcription factor-controlled pathways is unknown. Using a MYC-induced murine lymphoma model as well as MYC-transformed human B cell lines, we found that PAX5 controls c-MYC protein stability and steady-state levels. This promoter-independent, posttranslational mechanism of c-MYC regulation was independent of ITAM/BCR activity. Instead it was controlled by another PAX5 target, CD19, through the PI3K-AKT-GSK3 $\beta$ axis. Consequently, MYC levels in B cells from CD19-deficient mice were sharply reduced. Conversely, reexpression of CD19 in murine lymphomas with spontaneous silencing of PAX5 boosted MYC levels, expression of its key target genes, cell proliferation in vitro, and overall tumor growth in vivo. In human B-lymphomas, CD19 mRNA levels were found to correlate with those of MYC-activated genes. They also negatively correlated with the overall survival of patients with lymphoma in the same way that MYC levels do. Thus, CD19 is a major BCR-independent regulator of MYC-driven neoplastic growth in B cell neoplasms.
\end{abstract}

\section{Introduction}

The concept of oncogene addiction (1) is particularly well validated in hematological malignancies. "Liquid" tumors are thought to depend on fewer genetic alterations and thus be more sensitive to drugs targeting abnormally expressed oncoproteins, the success of Gleevec in treating tumors bearing Bcr-Abl being the prime example $(2,3)$. Additionally, initiating oncogenes in many lymphomas and leukemias are easily identified as products of recurrent chromosomal translocations. For example, in human Burkitt's and some diffuse large B cell lymphomas (DLBCLs), the $\mathrm{t}(8 ; 14)$ translocation places c-MYC under the control of the immunoglobulin heavy chain $(\mathrm{IgH})$ gene enhancer $(4,5)$. A similar translocation has been identified in murine plasmacytomas (6).

Another protein strongly implicated in B cell neoplasms is paired box transcription factor 5 (PAX5). PAX5 controls B cell differentiation from the pro- $\mathrm{B}$ to the mature $\mathrm{B}$ cell stage and is chiefly responsible for expression of the B cell receptor (BCR) complex $(7,8)$. This is achieved via direct transcriptional activation of genes encoding CD79a (also known as Ig- $\alpha$ ) (9), which heterodimerizes on the cell surface with CD79b (also known as Ig- $\beta$ ) (10) and the CD19 coreceptor $(11,12)$. Furthermore, a large body of genetic evidence also implicates PAX5 function in B-lymphomagenesis and leukemogenesis. The corresponding gene is affected by a relatively rare $(13,14)$ but persistent $\mathrm{t}(9 ; 14)(\mathrm{p} 13 ; \mathrm{q} 32)$ translocation (15-17) associated with aggressive B cell non-Hodgkin's lymphomas (NHLs) (18). In addition to genomic rearrangements, the PAX5 gene is also affected by somatic hypermutations, in particular in patients with DLBCL (19).

Somewhat unexpectedly, there are several other recurrent translocations (e.g., t[7;9][q11;p13] and t[9;12][q11;p13]) involving PAX5, which were found in B cell acute lymphocytic leukemia (B-ALL).

Conflict of interest: The authors have declared that no conflict of interest exists. Citation for this article: J Clin Invest. 2012;122(6):2257-2266. doi:10.1172/JCI45851.
These translocations result in the fusion of the Pax5 and ELN and ETV6/TEL genes $(20,21)$ and are regarded as dominant-negative inhibitors of PAX5 transcriptional activity. Furthermore, the genome-wide analysis of B-ALL using high-resolution SNP arrays and direct genome sequencing yielded several loss-of-function mutations in PAX5 (22). One possible way to reconcile the oncogenic and tumor suppressor activities of PAX5 is to posit that PAX5 affects neoplastic growth in a manner depending on stage differentiation and, in particular, on BCR expression. As most NHLs are derived from mature and pregerminal and postgerminal center $B$ cells, they express this growth-promoting complex $(23,24)$. In contrast, the vast majority of B-ALLs (A1 and A2 types) are derived from immature pro- or pre-B cells lacking BCR (25). If the transforming activity of PAX5 was dependent on BCR signaling, it would only manifest itself in NHL but not B-ALL, in which the intrinsic growth suppressive effects of PAX5 might be unmasked (26).

Indeed, our previous data demonstrate that induction of BCR signaling is important for MYC-induced PAX5-mediated lymphomagenesis (27). Specifically, PAX5-dependent neoplastic growth could be reduced by overexpression of immunoreceptor tyrosine-based activation motif-specific (ITAM-specific) CD22 phosphatase or treatment with Syk inhibitors or mimicked by forced expression of the constitutively active ITAM construct (28). Subsequent data using transgenic mouse models validated the idea that MYC and BCR signaling pathways cooperate during B-lymphomagenesis (29). Yet it remained unclear whether there is a direct functional interaction between the 2 key transcription factors, MYC and PAX5. We addressed this question using 2 cell models. One is the MYC5 cell line, derived by us from p53-null/MYC-induced murine lymphoma cells, which undergo spontaneous silencing of PAX5 when cultured in vitro $(27,30-33)$. The other is the P493-6 human B-lymphoblastoid cell line, immortalized by Epstein-Barr virus and expressing the MYC transgene from a tet-regulated promoter (34). In vivo and in 
A

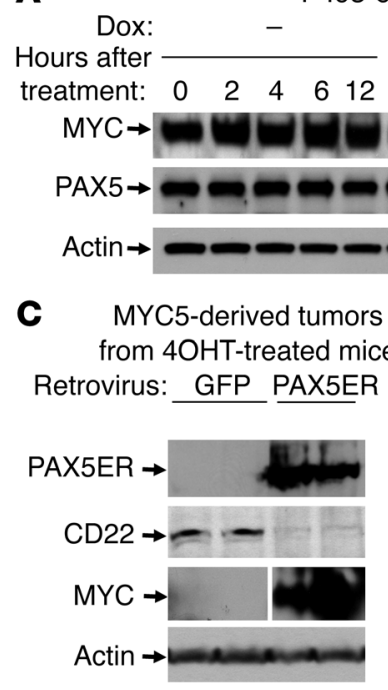

493-6 cells

$+$

$-+-+-+$

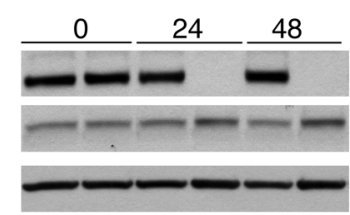

D

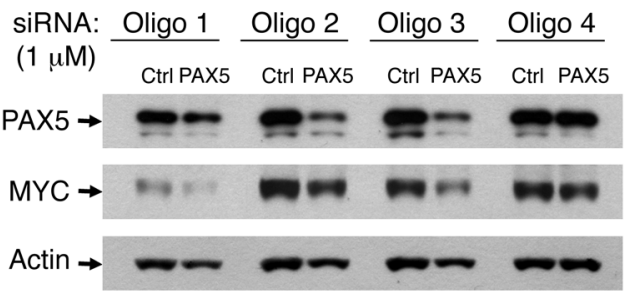

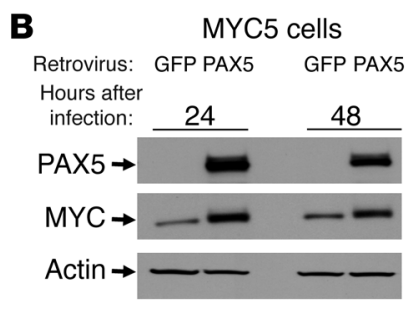

E

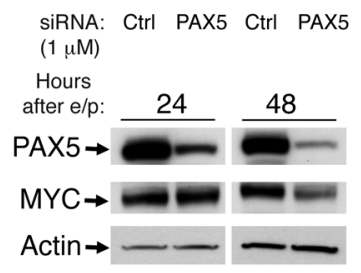

Figure 1

PAX5 regulates C-MYC protein levels. All panels represent immunoblotting analyses of proteins indicated. Actin was used as a loading control. (A) P493-6 cells either untreated (0 hours) or treated with Dox for indicated intervals prior to harvesting. (B) MYC5 cells infected with either the empty MIGR1 vector (GFP) or PAX5-MIGR1 (PAX5). Protein lysates were prepared 24 and 48 hours after infection. (C) Protein lysates were obtained from MYC tumors samples described previously (27). Prior to implantation, MYC5 cells were infected with either the empty MIGR1 vector or PAX5ERMIGR1 (PAX5ER). (D) P493-6 cells electroporated using various anti-PAX5 (PAX5) or control (Ctrl) siRNAs. (E) P493-6 cells were electroporated $(\mathrm{e} / \mathrm{p})$ with $1 \mu \mathrm{M}$ of either control or anti-PAX5 siRNA, and lysates were harvested 24 and 48 hours after electroporation.

vitro data obtained using these cell lines as well as primary murine $B$ cells and human NHLs yielded a surprising conclusion. We discovered that in B cells PAX5 and its downstream effector CD19 are important posttranscriptional regulators of MYC protein levels, with important implications for B-lymphomagenesis.

\section{Results}

Pax5 sustains c-Myc protein levels. We first asked whether shutting down MYC expression would compromise PAX5 protein levels. We treated P493-6 cells with doxycycline (Dox) for 2 to 12 hours. While MYC protein levels decreased sharply 2 hours after Dox treatment, there were no appreciable changes in PAX5 levels, as judged by Western blotting (Figure 1A, left). Even after prolonged exposure to Dox (24-48 hours), PAX5 expression levels were unaffected (Figure 1A, right). To determine whether changes in PAX5 expression affect MYC protein levels, we restored PAX5 expression in MYC5 cells (see Introduction) using the PAX5-encoding retroviral vector (MIGR1). Cells lysates were harvested 24 and 48 hours after infection and analyzed by Western blotting. At both time points, a clear increase in MYC levels was observed (Figure 1B). To determine whether MYC levels are induced by PAX5 in vivo, we analyzed MYC5 tumors reconstituted with conditionally active PAX5-estrogen receptor (PAX5ER) fusion (27). To ensure PAX5 activity, all tumor-bearing mice were treated with the ER ligand 4-hydroxytamoxifen (4OHT). In these specimens, there was a strong positive correlation between MYC and PAX5ER levels (Figure 1C). In contrast, CD22 levels were downregulated by PAX5, consistent with our previous finding (27).

To reproduce this result in a different cell system using a loss-offunction approach, we chose to knock down PAX5 with siRNA in P493-6 cells. To optimize transfection conditions, FITC-labeled, double-stranded RNA duplexes were electroporated separately into P493-6 cells, and flow cytometry was performed to determine the efficiency of siRNA delivery. At doses of $0.1 \mu \mathrm{M}$ and $1 \mu \mathrm{M}$ siRNA, transfection efficiency ranged between $62 \%$ and $96 \%$ (Supplemental Figure 1A; supplemental material available online with this article; doi:10.1172/JCI45851DS1). Then, increasing amounts of $\alpha$-PAX5 SMARTpool siRNA (10 nM, $0.1 \mu \mathrm{M}$, and $1 \mu \mathrm{M})$ were introduced into P493-6 cells, and protein lysates were harvested 24 hours after electroporation for Western blot analysis. We observed a sequence-specific, dosage-dependent decrease in PAX5 protein levels, which led to a commensurate decrease in CD19, a direct PAX5 target, with maximum inhibition of both proteins achieved at a dose of $1 \mu \mathrm{M}$ siRNA (Supplemental Figure 1B). To rule out off-target effects, we also analyzed 4 individual siRNAs comprising the SMARTpool. As expected, 3 out of 4 siRNAs inhibited PAX5 expression, and all 3 also caused a reproducible reduction in MYC levels (Figure 1D). Next, we treated the cells with $1 \mu \mathrm{M}$ of $\alpha$-PAX5 siRNA and assessed MYC levels 24 and 48 hours after electroporation. While no changes in MYC levels were apparent at 24 hours, they were appreciably reduced after 48 hours (Figure 1E). This result was suggestive of an indirect mechanism of regulation, presumably mediated by PAX5 targets.

$C D 19$, but not BCR, signaling increases c-Myc protein stability. Due to the absence of PAX5, MYC5 cells don't express PAX5 target genes, most notably CD79a. It is, however, robustly induced upon PAX5 reexpression (27) and can form a complex with constitutively expressed CD79b, resulting in phosphorylation of their ITAM motifs and BCR signaling. To determine whether this sequence of events increases MYC levels, we used MYC5 cells transduced with the MIGR1 retrovirus encoding the HA-tagged ITAM of CD79a/b in either a constitutively active (MYC5-ITAMwt cells) or a mutant configuration (MYC5-ITAMmut cells), in which both tyrosines are replaced with alanines (28). Infection efficiency was determined by flow cytometry, and transduced cells were 


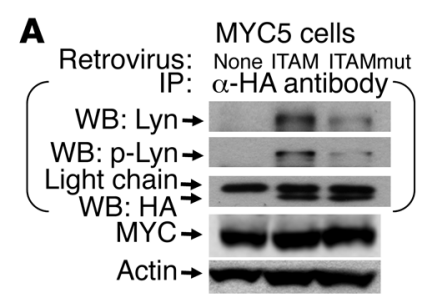

B

B MYC5 cells

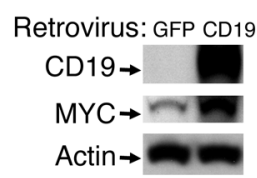

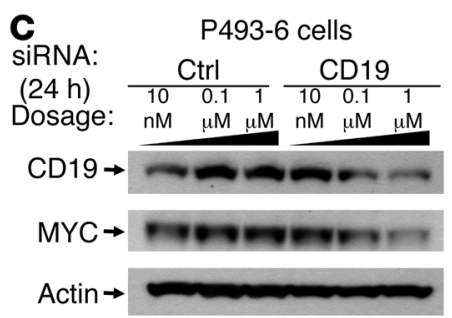

F

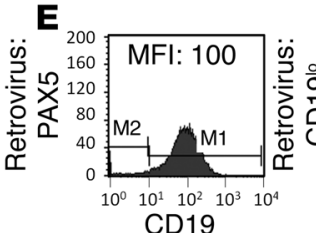

G

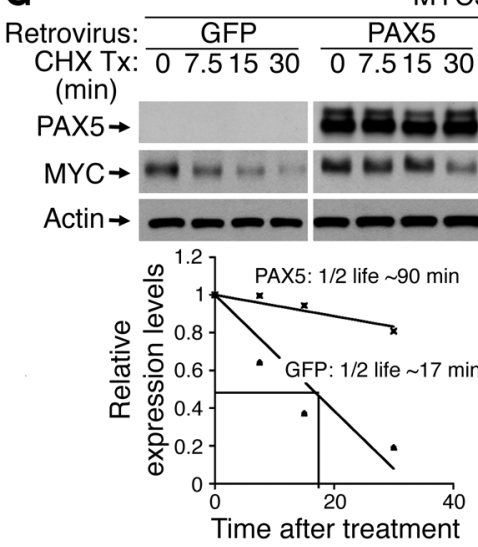

(min)

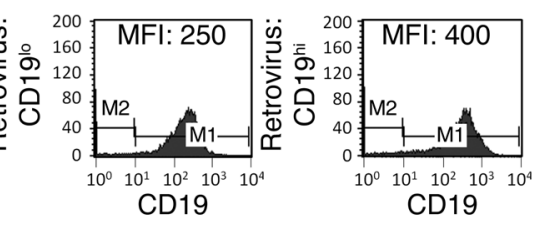

MYC5 cells

GFP
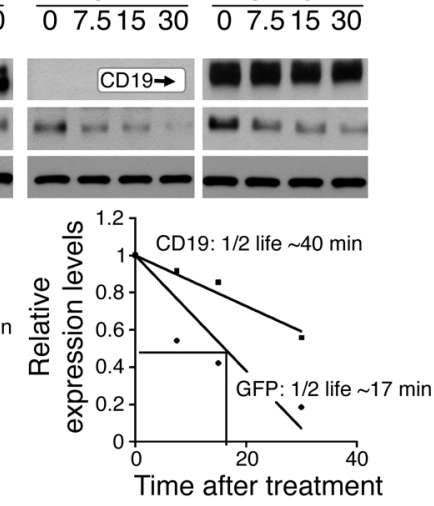

(min)
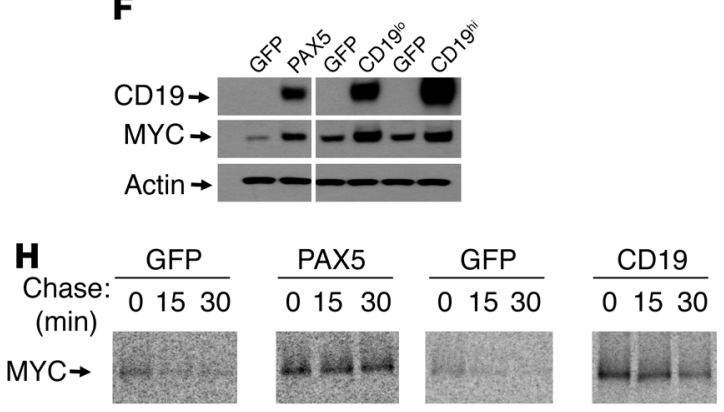

Figure 2

PAX5 regulates C-MYC protein levels through CD19. All panels except $\mathbf{E}$ and $\mathbf{H}$ represent immunoblotting analyses of proteins indicated. Actin was used as a loading control. (A) Whole cell lysates from MYC5 cells expressing HA-tagged wild-type or mutant ITAM (ITAMmut) were subjected to immunoprecipitation with $\alpha-\mathrm{HA}$ antibody, followed by immunoblotting with antibody against total Lyn, Tyr396-phospho-Lyn, or another $\alpha$-HA antibody (bracketed panels). Lysates from the same cells were immunoblotted with antibodies against C-MYC and $\beta$-actin (bottom panels). (B) CD19 and MYC levels in MYC5 cells transduced with empty vector (GFP) or CD19-encoding retrovirus (CD19). (C) PAX5, CD19, and MYC levels in P493-6 cells electroporated with increasing concentrations of control or $\alpha$-CD19 siRNA. (D) CD19 and MYC levels in bone marrow-derived B cells from wild-type and CD19-null (KO) mice. (E) Flow cytometric detection of CD19 expression on the surface of MYC5 cells expressing PAX5 or low/high levels of CD19. Mean fluorescent intensities are shown in each plot. (F) CD19 and MYC protein expression in the same cultures. (G) MYC levels in PAX5- and CD19-reconstituted cell lines after treatment with CHX. CHX was added at the concentration of $1 \mu \mathrm{g} / \mathrm{ml}$ for 7.5 to $30 \mathrm{minutes}$. MYC-specific bands were quantitated by densitometry, normalized to actin, and plotted against time after treatment. Note that different exposures are shown for parental and PAX5/CD19-transduced cells to allow accurate band quantitation. Symbols represent individual timepoints. (H) Steady-state MYC levels in the same cultures, detected by radioimmunoprecipitation. Cells were pulse-labeled with ${ }^{35} \mathrm{~S}-\mathrm{methionine}$ for 30 minutes, followed by a 0 to 30 minutes chase with "cold" amino acids.

confirmed to be more than $97 \% \mathrm{GFP}^{+}$(data not shown). Protein lysates from MYC5-ITAMwt and MYC5-ITAMmut cells were first subjected to immunoprecipitation with a mouse anti-HA antibody. Then immunoprecipitates were immunoblotted for the Src family kinase Lyn, which binds specifically to the ITAM of CD79a in B cells (35). Rabbit anti-HA antibody-reactive ITAM was used as a loading control. As anticipated, overexpression of wild-type ITAM (ITAMwt) led to an increase in Lyn binding compared with that of parental and mutant ITAM-transduced (ITAMmuttransduced) cells (Figure 2A). Additionally, the amount of ITAM-bound Lyn activated via phosphorylation on $\mathrm{Tyr}^{396}$ was also increased in ITAMwt cells. Of note, while ITAMmut induced some phospho-Lyn recruitment, it was not as robust as recruitment of Lyn by ITAMwt, attesting to the functionality of the
ITAM construct (Figure 2A). Still, there was no change in MYC protein levels (Figure 2A), indicating that activation of ITAM, a critical component of the BCR pathway, is insufficient to boost MYC protein expression.

Another major target of PAX5 is CD19 $(11,12)$. Although CD19 is thought to function in the context of $\mathrm{BCR}$, we considered the possibility that it might have BCR-independent functions, e.g., regulation of MYC. Thus, we transduced CD79a-negative MYC5 cells with a CD19 retrovirus and measured steady-state MYC levels. Surprisingly, MYC expression was increased several fold (Figure $2 \mathrm{~B}$ ). To reproduce this result in a different cell system using the loss-of-function approach, we knocked down CD19 in P493-6 cells using SMARTpool siRNA. Despite only partial knockdown of CD19, MYC levels were appreciably and consistently reduced 
A

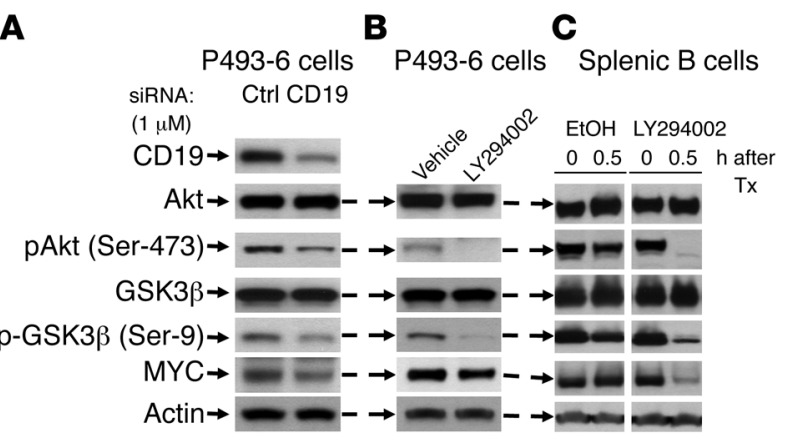

E

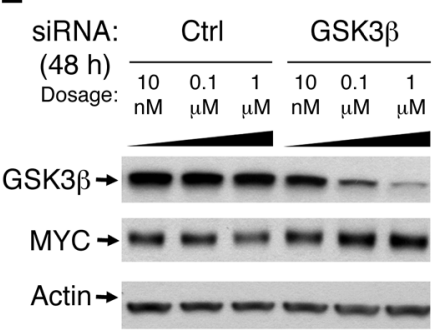

D

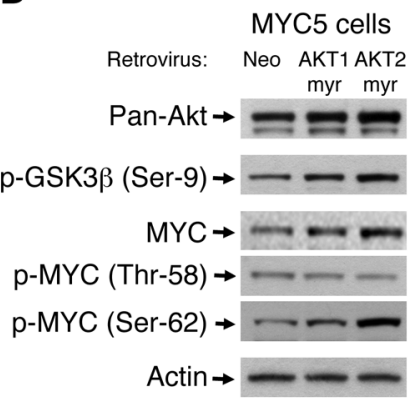

F

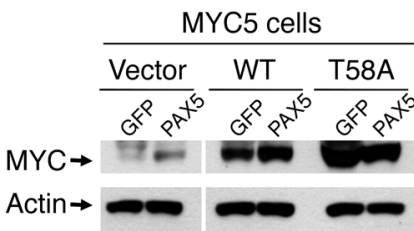

G

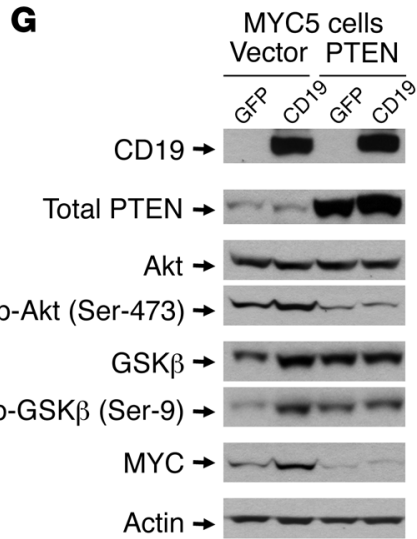

\section{Figure 3}

CD19 regulates MYC protein expression through the PI3K/AKT pathway. All panels represent immunoblotting analyses of proteins that belong to the CD19/PI3K pathway. Actin was used as a loading control. (A) P493-6 cells electroporated 24 hours prior to harvesting with $1 \mu \mathrm{M}$ of control or anti-CD19 siRNA. (B) The same cells treated with $10 \mu \mathrm{M}$ of PI3K inhibitor LY29004 or vehicle alone (ethanol) for 1 hour. (C) Splenic B cells pretreated with the same reagents for 0.5 hours. (D) MYC5 cells transduced with either empty retroviral vector (neo) or retroviruses expressing constitutively active forms of Akt1/2 (AKTmyr). Protein lysates were additionally probed with antibodies against MYC residues Thr-58 and Ser-62. (E) P493-6 cells electroporated 48 hours prior to harvesting with increasing concentrations of control or anti-GSK3 $\beta$ siRNA. (F) MYC5 cells expressing GFP or PAX5 were additionally transduced with either empty vector, WT MYC, or the T58A variant. Transduced cells were immunoblotted for MYC, and MYC protein levels in PAX5-transduced cells were compared with those in GFP-transduced cells. Note that the T58A variant has the Py-tag and thus migrates slower in SDS-PAGE gels. (G) MYC5 cells expressing GFP or CD19 from Figure 2B were additionally transduced with either empty vector or the PTEN-encoding retrovirus (PTEN).

while PAX5 levels were unaffected (Supplemental Figure 1C). We also analyzed individual siRNAs comprising the SMARTpool. The oligonucleotide responsible for most robust downregulation of CD19 also downregulated MYC in sequence-specific and dosedependent manners (Figure 2C). Finally, we measured MYC levels in bone marrow-derived B cells from CD19-deficient mice (36). To this end, short-term cultures of primary B cells were established. In these cultures, over $95 \%$ of cells were of B cell lineage, as confirmed by B220 positivity (Supplemental Figure 2A). Once again, CD19-deficient B cells contained lower MYC levels than their CD19-sufficient counterparts (Figure 2D). Of note, in the 3 models tested (MYC5, P493-6, and primary murine B cells), MYC expression was driven by 3 different regulatory elements (retroviral LTR, CMV IE region, and the endogenous myc gene promoter, respectively). Thus, the uniform downregulation of MYC upon CD19 silencing was indicative of promoter-independent, posttranscriptional regulation.

To address this hypothesis, we measured MYC protein halflife in MYC5 cells transduced with empty vectors compared with that in PAX5- and CD19-transduced MYC5 cells. For this experiment, CD19-positive cells were additionally fractionated into $\mathrm{CD} 19^{\text {lo }}$ and $\mathrm{CD} 19^{\mathrm{hi}}$ subpopulations. The levels of CD19 overexpression in these cultures were 2.5 and 4.0 fold, respectively, compared with PAX5-driven expression levels (see mean fluorescent intensities in Figure 2E). These calculations were confirmed by Western blotting. Nevertheless, MYC levels were comparable between CD19lo and CD $19^{\text {hi }}$ cultures, suggesting that 4-fold CD19 overexpression of CD19 is not required for MYC activation (Figure 2F).

Then PAX5- and CD19-reconstituted lines were treated with the protein synthesis inhibitor cycloheximide (CHX) (37), and steadystate MYC levels were measured by Western blotting. CHX treatment over the course of 30 minutes had no observable effects on retrovirally transduced PAX5 or CD19 proteins expression, but MYC levels were clearly downregulated (Figure 2G). However, while the half-life of MYC was approximately 17 minutes in vectortransduced MYC5 cells, MYC half-life was strongly increased, to approximately 90 and 40 minutes, respectively, in cells transduced with PAX5 or CD19 (Figure 2G).

CHX treatment has the potential to indirectly inhibit gene expression through changes in mRNA stability or protein translation. Thus, we also measured the half-life of MYC using pulselabeling followed by chase and radioimmunoprecipitation. Fully consistent with the results of the CHX experiment, MYC was bare- 
A

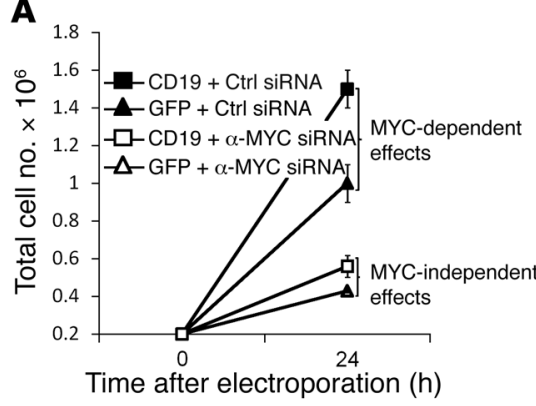

D

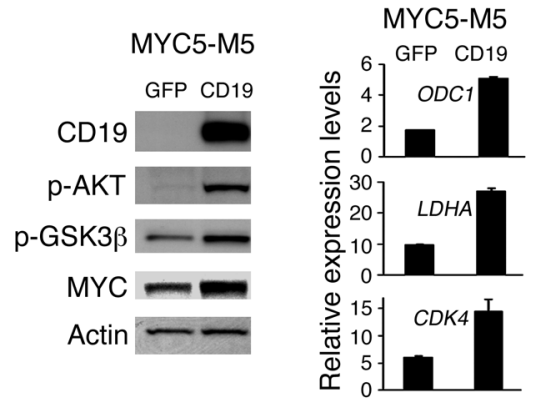

B

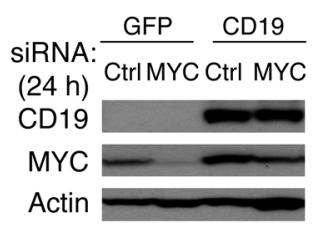

C

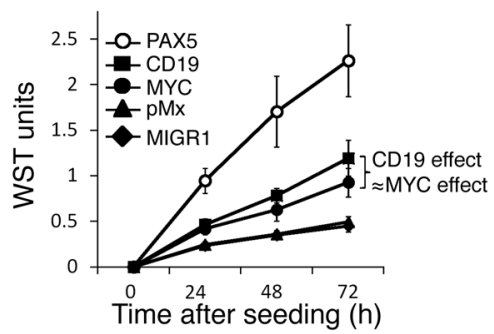

G

F

MYC5-M5 tumors

$\frac{\text { GFP }}{\text { T1 T2 T3 T4 T5 T6 }}$

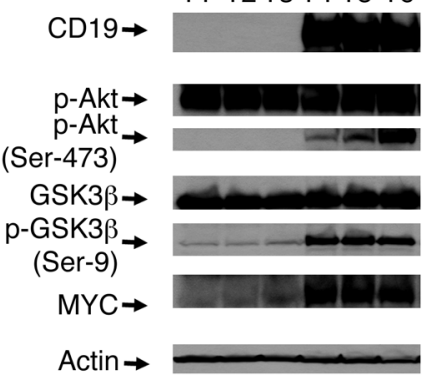

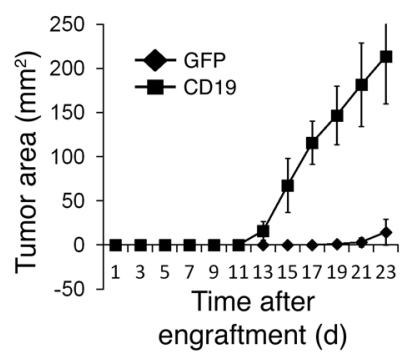

engraftment (d)

Figure 4

CD19 promotes cell expansion in vitro and tumor growth in vivo. Cell lines used in these experiments were either (A-C) MYC5 or (D-G) its single-cell subclone MYC5-M5. (A) Comparative analysis of growth rates of control- and anti-MYC siRNA-treated GFP- and CD19-reconstituted MYC5 cultures. (B) MYC protein levels in the same cultures. (C) Comparative analysis of growth rates of PAX5-, CD19-, and MYC-reconstituted MYC5 cultures. pMx, retroviral vector pMx-IRES-GFP; WST, absorbance at $440 \mathrm{nM}$ of media conditioned by cells treated with water-soluble tetrazolium salts. The effect of MYC on cell proliferation was approximately equal to that of CD19. (D) Expression levels of the CD19-MYC axis component in CD19-reconstituted MYC5-M5 cells. (E) Levels of known MYC target genes in cultures from D. (F) Expression levels of the CD19MYC axis component in CD19-reconstituted MYC5-M5 tumors after subcutaneous engrafting in SCID mice. Three individual tumors (T) from each cohort were randomly chosen for this analysis. (G) Growth rates of tumor xenografts from $\mathbf{F}$. No less than 5 mice were analyzed in each group. Error bars denote standard deviations.

ly detectable after approximately 30 minutes of chase in parental cells but was strongly stabilized in the presence of either PAX5 or CD19 (Figure 2H). Thus, these 2 proteins are indeed positive posttranscriptional regulators of MYC protein levels.

$B C R$-independent $A K T$ activation is necessary and sufficient for $M y c$ induction by $C D 19$. One of the known functions of CD19 signaling is the recruitment and activation of PI3K (38) and ensuing activation of Akt (39-42), all in the context of BCR signaling (reviewed in ref. 43). To determine whether Akt activation accompanies the stabilization of MYC by CD19, we electroporated P493-6 cells with anti-CD19 siRNA and assessed expression levels of the Akt signaling components. As shown in Figure 3A, CD19 knockdown decreased not only MYC protein levels but also those of phosphoserine-473 in Akt and phosphoserine-9 in Akt's target GSK3 $\beta$. Total Akt and GSK3 $\beta$ levels were unchanged. To determine whether Akt phosphorylation was necessary for MYC stabilization, we treated P493-6 cells with the PI3K inhibitor LY294002. As anticipated, 1-hour LY294002 treatment resulted in decreased pAkt and pGSK3 $\beta$ levels as well as decreased MYC levels (Figure 3B). Since the reduction in MYC levels was relatively modest, we also repeated this experiment in splenic B cells (obtained as described in Methods) and analyzed for purity using flow cytometry (Supplemental Figure 2B). These short-term cultures responded to LY294002 very robustly, as evidenced by very low pAkt and PGSK3 $\beta$ levels and sharply downregulated MYC (Figure 3C).
To further establish the role of Akt in MYC induction, we transduced MYC5 cells with retroviruses encoding constitutively active Akt1 and Akt2. In both cases, especially in Akt2-transduced cells, we observed a commensurate increase in inhibitory GSK3 $\beta$ phosphorylation and MYC steady-state levels (Figure 3D). In addition to being an Akt target, GSK3 $\beta$ negatively regulates MYC levels by phosphorylating Thr-58, provided that Ser-62 is already phosphorylated by another kinase (reviewed in refs. 44,45 ). This allows binding of the $\mathrm{Fbw} 7$ ubiquitin ligase, which contributes to MYC degradation (46). Thus, the same protein lysates were probed with antibodies against MYC residues Thr-58 and Ser-62. While Thr-58 phosphorylation was decreased in Akt-expressing cells, GSK3 3 -independent Ser-62 phosphorylation was increased, consistent with published data (47).

To determine whether GSK3 $\beta$ controls MYC protein levels in our model system, increasing concentrations of anti-GSK3 $\beta$ siRNA $(10 \mathrm{nM}, 0.1 \mu \mathrm{M}$, and $1 \mu \mathrm{M})$ were electroporated into P493-6 cells. As evidenced by data in Figure 3E, GSK3 $\beta$ knockdown led to robust upregulation of MYC, attesting to the key role of the $\mathrm{PI} 3 \mathrm{~K} / \mathrm{Akt} / \mathrm{GSK} 3 \beta$ pathway in regulating MYC protein output in B cells. Similar effects were observed with individual siRNA comprising the SMARTpool (see Supplemental Figure 1D and data not shown.) To further validate this finding, we analyzed the effects of mutating the GSK3 $\beta$ consensus site in MYC (Thr58Ala). GFP- and PAX5-overexpressing MYC5 cells were additionally 
A

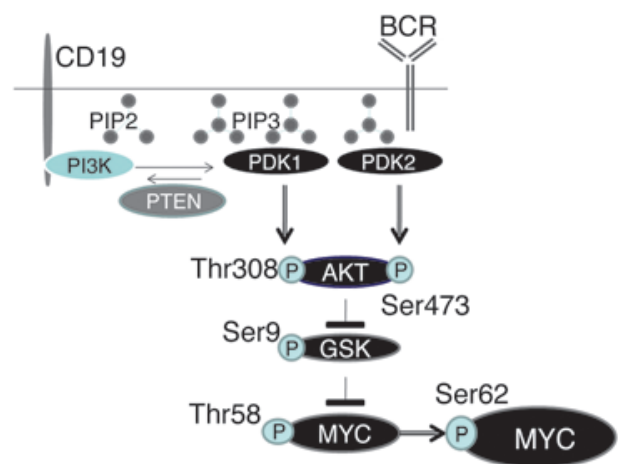

B

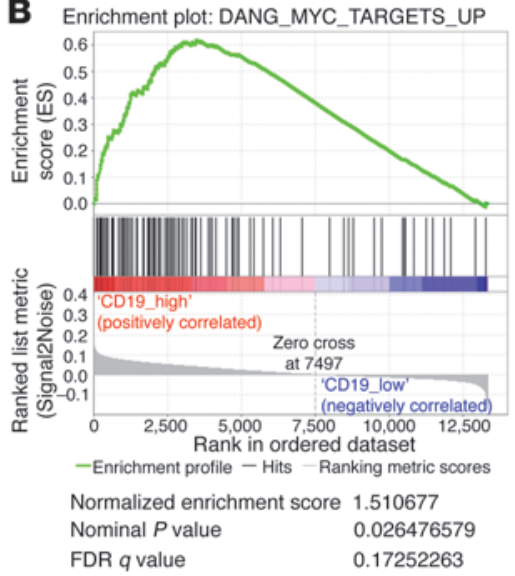

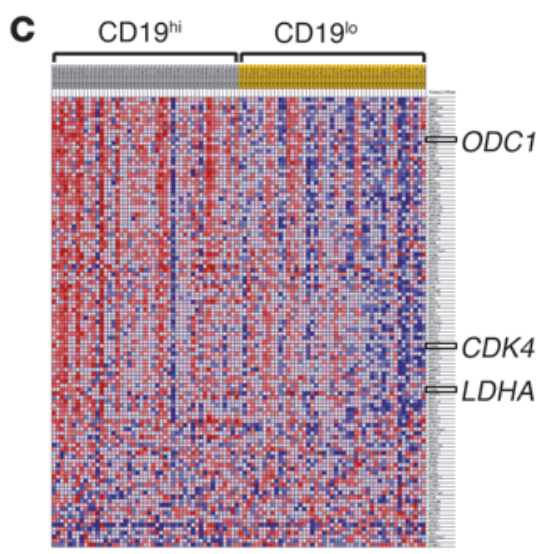
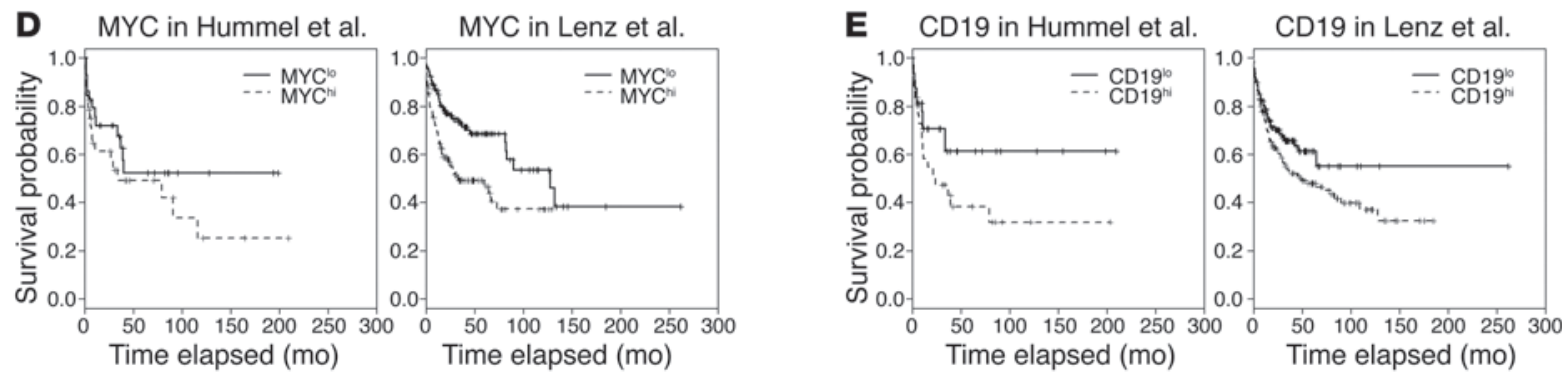

Figure 5

CD19 contributes to MYC function in human B-lymphomas. (A) Model wherein CD19 promotes MYC stabilization in a BCR-independent manner (see Results for more explanation). (B) GSEA enrichment plot for the DANG_MYC_TARGETS_UP set comparing CD19hi tumors with CD19lo tumors. The normalized enrichment score, $P$ value, and FDR $q$ value are indicated below the plot. (C) Heat map generated during GSEA depicting the MYC target genes and comparing CD19hi tumors with CD19lo tumors. (D) KM curves comparing survival of MYC ${ }^{\text {hi }}$ patients with that of MYClo patients in the Hummel (53) and Lenz (57) studies. (E) KM curves comparing survival of CD19hi patients with that of CD19lo patients in the Hummel (53) and Lenz (57) studies.

transduced with either wild-type or T58A MYC constructs. We observed that PAX5 increased expression levels of both endogenous and retrovirally encoded wild-type MYC but not the T58A variant (Figure 3F).

Finally, we sought to determine whether the Akt-GSK3 $\beta$ axis is necessary for MYC activation by CD19 in BCR-negative cells. The analysis of control vector-transduced (MSCV-ires-NGFRtransduced) and CD19-transduced MYC5 cells revealed the same increase in activating phosphorylation of Akt and inhibitory phosphorylation of GSK3 $\beta$ as in BCR-sufficient B cells (Figure 3G, left 2 columns). Then we repeated the same experiment with cells engineered to overexpress phosphatase and tensin homolog (PTEN), the key negative regulator of the PI3K pathway. As expected, PTEN completely abolished Akt activation by CD19 and also prevented MYC upregulation (Figure 3G, right 2 columns), establishing a causal relationship between these 2 phenomena.

The CD19-Myc axis promotes cell expansion in vitro and in vivo. Given the prominent role of MYC in cell division, we next asked whether CD19 promotes cells growth in a MYC-dependent manner. To this end, we treated parental and CD19-expressing MYC5 cells with siRNA against MYC, bringing down both basal and CD19induced MYC levels. As expected, the effects of CD19 on cell growth in the presence of $\alpha$-MYC siRNA were much smaller than those seen in the presence of control siRNA (Figure 4A). However, in the presence of CD19, anti-MYC siRNA was not as effective as in control MYC5 cells in reducing MYC levels (Figure 4B), complicating interpretation of the results. Thus, we also directly compared growth rates of PAX5-, CD19-, and MYC-transduced MYC5 cells (from Figure 2F and Figure 3F, respectively). PAX5transduced cells grew appreciably faster when compared with cells transduced with CD19 or MYC (Figure 4C), perhaps because, in addition to CD19, PAX5 reactivates CD79a and growth-promoting BCR signaling (27). However, CD19- and MYC-transduced cells proliferated at approximately the same rates, suggesting that CD19-induced proliferation doesn't have a significant MYC-independent component (Figure 4C).

To determine whether CD19 also promotes cell growth in vivo, we used the MYC5-M5 subclone, which doesn't reactivate PAX5 and CD19 when injected into mice $(27,30)$. Reconstitution of MYC5-M5 cells with CD19 was performed as described for parental MYC5 cells, using sorting for GFP (Supplemental Figure 3A). As anticipated, MYC5-M5/CD19 cells retained their quasi-myeloid lineage, in that they were still positive for Mac1 and negative for B220 (Supplemental Figure 3B). They also robustly upregulated phospho-Akt and MYC (Figure 4D). To determine whether MYC target genes responsible for cell cycle progression are upregulated in CD19-reconstituted cells, we measured mRNA levels of 3 key MYCactivated genes, $O D C 1(48), \operatorname{LDHA}(49,50)$, and $C D K 4$ (51). In all 3 cases, we observed elevated mRNA levels in CD19-sufficient cultures compared with those in CD19-deficient cultures (Figure 4E). Also, we had previously demonstrated that widespread downregulation of microRNAs by MYC contributes to neoplastic growth 
(52). Thus, we compared levels of 5 key MYC-repressed microRNAs in CD19-negative and -reconstituted MYC5 cells lines. For all 5 growth-suppressive microRNAs tested (miR-16, miR-34a, miR-150, miR-195, and let-7e), we observed decreased levels in CD19-reconstituted cells (Supplemental Figure 3C).

Control and CD19-reconstituted MYC5-M5 cells were injected into immunocompromised mice. The resultant tumors were analyzed for the components of the CD19-MYC axis. As anticipated, increased MYC levels were accompanied by increased levels of phospho-Akt and phospho-GSK3 $\beta$ (Figure 4F), while tumor cells expressing mutant CD19 failed to activate Akt and upregulate MYC protein expression, further stressing the importance of this pathway for MYC-dependent neoplastic growth. Of note, CD19-reconstituted cells grew much faster and ultimately formed much larger neoplasms (Figures 4G) than control GFP-only cells. This allowed us to propose a molecular model wherein CD19 controls MYC levels in a manner dependent on PI3K but not the BCR (Figure 5A).

Finally, we asked whether CD19 contributes to MYC function in human B-lymphomas. We hypothesized that tumors exhibiting increased CD19 expression would in turn exhibit increased expression of MYC targets. To this end, we analyzed the publicly available data set corresponding to hundreds of tumor specimens profiled in the Hummel study (53). To avoid Burkitt's lymphoma cases that frequently harbor Thr-58 mutations (54), we focused on 142 DLBCLs without Ig-MYC translocations. Using these filtered cases, we categorized patient samples into 3 groups (CD19 high, intermediate, and low) and performed gene set enrichment analysis (GSEA) (55) comparing CD19hi and CD19lo tumors. We observed that CD19hi tumors exhibited enrichment for the curated gene set DANG_MYC_TARGETS_UP (ref. 56 and Figure 5B). This enrichment was highly significant, with a normalized enrichment score of 1.510677 , a $P$ value of less than 0.026476579 , and a false discovery rate (FDR) $q$ value of less than 0.17252263 . Importantly, the enriched set included the 3 well-validated MYC targets ODC1, $C D K 4$, and $L D H A$, as demonstrated in the heat map in Figure 5C.

Since this analysis strongly connects CD19 expression to the expression of MYC targets, we further postulated that CD19 and MYC would have similar effects on patient survival. In addition to the Hummel analysis, we included the Lenz study, which profiled mRNA expression in conjunction with survival data for hundreds of patients with DLBCL (57). In order to maintain the fidelity of each individual study while gaining statistical insight from both, we used the stratified log-rank test to determine the significance of gene expression levels for overall survival. Of note, the distribution of normalized MYC and CD19 values and the estimated KaplanMeier (KM) curves of the 2 studies were similar, confirming the appropriateness of combining the 2 studies. We first investigated how MYC levels would affect patient survival. In each study, the patients with low MYC expression values had better survival, compared with that of patients with high MYC values (Figure 5D). The stratified log-rank test yielded a $\chi^{2}$ value of 15.17 and a $P$ value of less than 0.0001 , suggesting a significant negative effect of MYC on survival. We next explored how CD19 affects patient survival. Confirming our hypothesis, CD19 expression and MYC expression had similar effects on patient survival (compare Figure 5, D and E). Again, the stratified log-rank test (58) yielded a $\chi^{2}$ value of 5.39 and a $P$ value of 0.0203 , suggesting that CD19 levels have a significant negative effect on patient survival. Thus, both the GSEA and survival analysis provide evidence that CD19 positively regulates MYC function in human B-lymphomas.

\section{Discussion}

The role of PAX5 in promoting B-lymphomagenesis is now well recognized (26). Key evidence includes the relatively rare $(14,18)$ but persistent $\mathrm{t}(9 ; 14)$ (p13; q32) translocation (15-17) juxtaposing PAX5 and the IgH locus; somatic hypermutations of PAX5 in DLBCLs (19) presumed to enhance PAX5 expression; and gene knockdown/overexpression studies performed in our laboratory (27). Negative effects on B cell expansion were also observed after knockdown of the PAX5 effector CD79a in DLBCL cells lines (24). Since CD79a is the central structural component of the BCR (8), these results suggested that stimulation of CD79a/BCR signaling by PAX5 underlies its transforming activity. Indeed, in our own study, we observed that constitutively active CD79a/b heterodimer (ITAM) also promotes B-lymphomagenesis (27). Similar conclusions regarding the role of BCR in neoplastic growth have been reached by other laboratories (29). Cooperation between BCR and MYC has been observed, hinting that they might be acting in parallel, not collinear pathways (29). Indeed, we report here that while restoration of PAX5 expression strongly augments MYC levels, ITAM has no appreciable effect on MYC levels. Instead this role could be ascribed to CD19-mediated signaling.

CD19 is a well-known B cell surface molecule, which, upon BCR activation, enhances B cell antigen receptor-induced signaling crucial for the expansion of the B cell population (59). One known mechanism of its action is the recruitment and activation of PI 3 and subsequently Akt kinases (38-42). This is accomplished through the docking function of 2 tyrosine residues, Y482 and Y513, in the cytoplasmic region of CD19 (60). Furthermore, inhibitory phosphorylation by Akt of GSK3 $\beta$ on Ser- 9 is one of the key functions of this pathway (61). In turn, phosphorylation of MYC on Thr-58 by GSK3 $\beta$ (reviewed in refs. 44, 62) leads to enhanced recognition by the Fbw7 E3 ubiquitin ligase and accelerated protein degradation (46). However, MYC protein stability is controlled extensively by other phosphorylation sites and other ubiquitin ligases (reviewed in refs. 44, 45). Hence, it was surprising to find that in B cells inactivation of GSK3 $\beta$ has such profound effects on MYC protein levels (see for example Figure 3E). The other surprise was that stimulation of Akt signaling by CD19 (as seen in Figure 3G) was observed in cells lacking the core BCR component CD79a and thus was completely independent of BCR signaling, which is known to control survival of mature B-lymphocytes (63-65).

Not only does CD19 augments MYC levels, it clearly has a positive effect on MYC function. For example, MYC5-M5 cells reconstituted with CD19 exhibited elevated levels of MYC targets ODC1, CDK4, and LDHA (Figure 4E). Moreover, in human DLBCLs there was a highly statistically significant correlation between CD19 mRNA levels and those of MYC-activated genes (Figure 5, B and $\mathrm{C})$, including $O D C 1, C D K 4$, and $L D H A$. Thus, it is not surprising that CD19 levels in DLBCL negatively correlated with patient survival, just like MYC levels do (Figure 5, D and E).

CD19 is broadly expressed in both normal and neoplastic B cells. Because B cell neoplasms frequently maintain PAX5 and CD19 expression, it (along with CD20) is regarded as the target of choice for a variety of immunotherapeutic agents, including immunotoxins $(59,66)$. In particular, humanized anti-CD19 mAbs and allogeneic $\mathrm{T}$ cells expressing chimeric antibody receptor for CD19 have entered clinical trials in recent months. They are presumed to work by recognizing and depleting CD19-expressing neoplastic B cells $(67,68)$, but the picture could be more complicated. Notably, treatment with anti-CD19 antibodies typically results in internalization of CD19 (69) and, by inference, loss of its function. Thus, 
in addition to delivering the anticancer drug or T cells, conjugated anti-CD19 antibodies are likely to reduce PI3K signaling and as a result compromise MYC expression. Whether this unintended consequence could be further exploited therapeutically represents an important area for future research.

Another important question is what drives cell proliferation in CD19-negative B cell neoplasms such as multiple myelomas (MMs), which are derived from plasma cells. Interestingly, MYC rearrangements are very common in this disease $(70,71)$, and recently described inhibitors of MYC gene transcription (JQ1) were used to demonstrate the essential role of MYC in MM pathogenesis (72). One can envision 2 explanations for how MYC levels are sustained in $\mathrm{MM}$ in the absence of CD19. One explanation is that its rearranged allele drives such robust transcription that the MYC protein can accumulate to sufficient levels even in the absence of PI3K signaling. The other more likely explanation is that in MM (and other CD19negative neoplasms such as Hodgkin's lymphoma) surface receptors other than CD19 are responsible for PI3K activation. This view is supported by the observation that at least in vitro MM cells are highly sensitive to PI3K inhibitors (73), possibly through destabilization of MYC, the key oncoprotein in neoplasms of B cell origin.

\section{Methods}

Cell cultures and treatment with P13K inhibitor. MYC5 and MYC5-M5 murine B-lymphoma cells were cultured as described previously $(27,30,31,74)$. The human B-lymphoblastic cell line P493-6 carries an inducible c-MYC repression system, in which c-MYC transcription is driven by the synthetic tetO7-TP promoter (34). They were cultured in RPMI-1640 (BioWhittaker) supplemented with $10 \%$ heat-inactivated tet-free FBS (Clontech), $100 \mathrm{U} / \mathrm{ml}$ penicillin, and $100 \mu \mathrm{g} / \mathrm{ml}$ streptomycin (Gibco). c-MYC expression in P493-6 cells was inhibited upon addition of Dox. In some experiments, P493-6 cells were treated with the PI3K inhibitor LY294002 (no. 9901; Cell Signaling Technology) at a concentration of $10 \mu \mathrm{M}$.

Primary B cells cultures. Whole spleens were obtained from C57BL/6 female mice (8-12 weeks of age) and gently ground with the round-ended plunger of a 1-ml syringe, followed by passing the cells over a 70-microncell strainer. Red blood cells from the splenocytes were lysed with red cell lysis buffer (Sigma-Aldrich) for 2 minutes at room temperature (RT). Cells were then counted and seeded at $1 \times 10^{6}$ cells $/ \mathrm{ml}$ in complete RPMI medium supplemented with $50 \mu \mathrm{M} \beta$-ME. After culturing for 2 hours in an incubator set at $37^{\circ} \mathrm{C}$ with $5 \% \mathrm{CO}_{2}$, floating cells were harvested. They were seeded at $1 \times 10^{6}$ cells $/ \mathrm{ml}$ with the aforementioned medium supplemented with LPS (stock concentration $10 \mu \mathrm{g} / \mathrm{ml}$ ) used at 1:1,000 dilution. Splenocytes were cultured for 3 days with LPS. Medium and LPS were replenished every day, and cells were also counted every day to check for growth. By day 3 , cells were more than $90 \% \mathrm{CD} 19^{+} \mathrm{B} 220^{+}$as accessed by flow cytometry. Bone marrow from female WT or CD19 knockout mice (36) (C57BL/6 background, 8-12 weeks of age) was obtained by flushing the femurs and tibias with complete DMEM. Red blood cells from the bone marrow were lysed with red cell lysis buffer (Sigma-Aldrich) for 2 minutes at RT. Cells were then counted and seeded at $0.5 \times 10^{6}$ cells $/ 35-\mathrm{mm}$ dish in MethoCult medium containing IL-7. Day 9 cells were more than $90 \%$ CD $19^{+}$B $220^{+}$ as accessed by flow cytometry. BD Pharmingen antibodies used for flow cytometry were PE rat anti-mouse CD19 (catalog no. 557399) and FITC rat anti-mouse CD45R/B220 (catalog no. 553088). Control antibodies include FITC rat $\operatorname{IgG}_{\mathrm{a}}$ (catalog no. 553929) and PE rat $\operatorname{IgG}_{\mathrm{a}}$ (catalog no. 553930).

Retroviral production and transduction. To overexpress PAX5 or CD19 in MYC5 cells, cells were transduced with retroviral construct PAX5-MIGR1 $(27,30)$ or CD19-pMx-ires-GFP (provided by Tomohiro Kurosaki at RIKEN Research Institute, Yokohama, Japan). Transduction with empty vector served as con- trol. To elucidate the effect of ITAM-mediated signaling in MYC5 cells, cells were transduced with the retrovirus MIGR1 encoding either the WT or the mutant form of ITAM, in which both tyrosines of the $\mathrm{Yxx}(\mathrm{L} / \mathrm{I}) \mathrm{x} 6-8 \mathrm{Yxx}(\mathrm{L} / \mathrm{I})$ motif were replaced with alanine. ITAM constructs were provided by John Monroe, Genentech, South San Francisco, California, USA. Transduced cells were analyzed for GFP expression 24 hours after transduction to determine transfection efficiency. Transduced cells were also sorted for GFP expression using identical gates based on GFP expression and collected for protein analysis. To overexpress the constitutively active form of AKT, the retroviral construct pLNCX1 encoding HA-tagged myristoylated AKT1 or AKT2 was transduced into MYC5 cells. Constructs were provided by Morris Bimbaum at the University of Pennsylvania. Transduced cells were selected with G418 (Sigma-Aldrich) for 2 weeks, and overexpression of retroviral constructs was assayed through HA expression by Western blotting. cDNAs of murine PTEN, WT human MYC, and the T58A MYC variant were cloned into the retroviral vector MSCV-ires-NGFR. The T58A MYC variant was provided by Michael Cole, Dartmouth Medical School, Hanover, New Hampshire, USA. Transfection efficiency was determined by flow cytometry with either isotype control antibody PE mouse IgG1 (555749, BD Pharmingen) or PE mouse anti-human CD271/NGFR (560927, BD Pharmingen) to detect the expression of NGFR on the cell surface. All retroviruses were generated using transfection with Lipofectamine 2000 (Invitrogen) into GP293 cells. Infections were carried over the course of 30 hours in the presence of polybrene $(4 \mu \mathrm{g} / \mathrm{ml})$.

Western blotting and immunoprecipitations. Total cell lysates were prepared, and Western blotting was performed as described previously (74). Antibody against PAX5 was provided by Michael L. Atchison, University of Pennsylvania. Other primary antibodies used in this study include anti-CD19 (no. 3574, Cell Signaling Technology), anti-PAN AKT (no. 4691, Cell Signaling Technology), anti-phospho-AKT (Ser-473; no. 4060, Cell Signaling Technology), anti-Total GSK3 $\beta$ (no. 9315, Cell Signaling Technology), antiphospho-GSK3 $\beta$ (Ser-9; no. 9336, Cell Signaling Technology), and antiTotal c-MYC (OP-10, Calbiochem) for detecting retroviral c-MYC in P493 and MYC5 cells; anti-Total c-MYC (no. 5605, Cell Signaling Technology) for detecting c-MYC in splenic B and bone marrow-derived B cells; and antip-c-MYC Thr-58 (A00242, Genscript), anti-p-c-MYC Ser-62 (ab78318, abcam), anti-Total Lyn (no. 2732, Cell Signaling Technology), anti-phospho-Lyn (tyr396; ab40660, Abcam), rabbit anti-HA clone (catalog no. H6908, SigmaAldrich), anti-CD22 (sc-7932, Santa Cruz Biotechnology Inc.), and anti$\beta$-Actin (A3853; Sigma-Aldrich). For immunoprecipitations, HA-tagged WT or mutant ITAM-transduced MYC5 cells were harvested by washing once in cold PBS, followed by lysis in cold passive lysis buffer containing protease and phosphatase inhibitors. HA-tagged ITAMs were immunoprecipitated from an equal number of cells for each sample at a cell-to-volume ratio of $1 \times 10^{6}$ cells $/ \mathrm{ml}$ of passive lysis buffer using a 1:1,000 dilution of the mouse monoclonal anti-HA clone 12CA5 (catalog no. 11583816 001; Roche). Antibody incubation was performed at $4^{\circ} \mathrm{C}$ for 14 to 18 hours, followed by immunoprecipitation with Protein A beads (Invitrogen) and washing with cold passive lysis buffer containing protease and phosphatase inhibitors.

CHX experiment. MYC5 cells stably transduced with either PAX5 or CD19 retroviral construct were seeded at $1 \times 10^{6}$ cells $/ \mathrm{ml}$ in $50-\mathrm{ml}$ conical tubes and received CHX (Sigma-Aldrich) treatment at $1 \mu \mathrm{g} / \mathrm{ml}$, while stimulation with 95\% ethanol (Sigma-Aldrich) served as control. Cells were stimulated for $0,7.5,15$, and 30 minutes and harvested by spinning at $275 \times \mathrm{g}$ for 1 minute, followed by immediate lysis in protein lysis buffer (Bio-rad). Protein samples were resolved on 10\% SDS-PAGE for Western blot analysis. c-MYC protein levels were quantified by densitometry using ImageJ 1.43 $(\mathrm{NIH})$, with quantitated values normalized to $\beta$-actin.

Pulse-chase experiment. MYC5 cells stably transduced with either PAX5 or CD19 retroviral construct were prestarved for methionine by replacing the culture medium with RPMI lacking L-methionine for 1 hour. Cells were 
labeled in vivo with ${ }^{35} \mathrm{~S}$-methionine using $0.2 \mathrm{mCi} / \mathrm{ml}$ at the density of $10^{7} \mathrm{cells} / \mathrm{ml}$ for 30 minutes. After labeling, cells were immediately washed once with RPMI containing $5 \mathrm{mM} \mathrm{L}$-methionine and then incubated in the same media for the indicated chase times. Cells were harvested and c-MYC proteins were immunoprecipitated. Labeled c-MYC was visualized by autoradiography and quantified using a phosphorimager.

Tumor load studies. For in vivo experiments, $1.5 \times 10^{7} \mathrm{MYC5}$-M5 cells were stably transduced with empty retroviral vector, $\mathrm{pMx}$-ires-GFP, or retroviral vector encoding CD19. Transduced cells sorted for the highest $\mathrm{GFP}^{+}$ expression were injected subcutaneously into SCID mice (National Cancer Institute). Tumor size was measured every other day using a caliper, and tumor weights were recorded on the day of tumor excision. Protein lysates were obtained from tumors for Western blot analysis by lysing tumors at a ratio of $40 \mathrm{mg}$ of tumor per $\mathrm{ml}$ of lysis buffer. Tumors from MYC5-M5 cells infected with retrovirus encoding PAX5ER ${ }^{\text {TAM }}$ (4OHT-treated) or its control vector (also 4OHT-treated) were obtained from a previous study (27), and protein lysates were obtained for Western blot analysis.

Proliferation assay. Proliferation of MYC5-M5 CD19-transduced cells in vitro was determined using cell proliferation reagent WST-1 (Roche Molecular Biochemicals). Cells were seeded at $1 \times 10^{3}$ cells per well in $100 \mu$ l of culture medium and were incubated for a total of 72 hours. Proliferation status was determined every 24 hours by incubating with $10 \mu$ l of the cell proliferation reagent WST- 1 for 4 hours. The absorbance of the treated samples in triplicates was measured against a blank control and was measured at $440 \mathrm{nM}$ using a Synergy 2 microplate reader (BioTek Instruments).

siRNA knockdown experiments. P493-6 cells were treated with the doublestranded ON-TARGET plus SMARTpool siRNA against human PAX5 (L-012241-00, Dharmacon), human total GSK3 $\beta$ (L-003010-00, Dharmacon), or human CD19 (sc-29968, Santa Cruz Biotechnology Inc.). A nontargeting pool of siRNA (D-001810-10, Dharmacon) was used as negative control for siRNA experiments. ON-TARGET sets of 4 individual siRNAs against human PAX5, CD19, or GSK3 $\beta$ were used. siRNA was electroporated into P493-6 cells with Amaxa Nucleofactor according to the manufacturer's instructions (Lonza). Efficiency of siRNA delivery was confirmed with a nonspecific double-stranded BLOCK-iT fluorescent oligo (no. 2013, Invitrogen). The effectiveness of silencing was confirmed by Western blotting. MYC5 cells stably transduced with CD19 retroviral construct or the control vector were electroporated with $10 \mathrm{nM}$ of control or anti-human MYC siRNA (L-003282, Dharmacon). Cells were harvested 24 hours after electroporation and counted.

Real-time q-PCR. Real-time q-PCR was performed with the 7500 Real-Time PCR System (Applied Biosystems). Primer sequences used for amplifications in murine MYC5-M5-derived tumors were as follows: CDK4 sense 5'-CAATGTTGTACGGCTGATGG-3'; CDK4 antisense 5'-CAGGCCGCTTAGAAACTGAC-3'; ODC1 sense 5'-GTGGCAACTCATGAAGCAGA-3'; ODC1 antisense 5'-TGCAGGCAAGAGCTACAAGA-3'; LDHA sense 5'-TCCGTTACCTGATGGGAGAG-3'; LDHA antisense 5'-GTAGGCACTGTCCACCACCT-3'.

Statistical analysis of cell and tumor growth. Cell proliferation study was performed in triplicates. The CD19 tumor study was performed with
5 mice per group. Statistical significance was assessed by a 1-tailed unpaired Student's $t$ test.

Gene set enrichment and survival analyses. Data from the Hummel (53) and Lenz (57) studies were downloaded from the Gene Expression Omnibus gateway (http://www.ncbi.nlm.nih.gov/geo/) using accession numbers GSE4475 and GSE10846, respectively. Patients from the Hummel study were initially sorted as Ig-MYC translocation positive and negative. The translocation-negative neoplasms were further sorted as CD19 high, intermediate, or low. GSEA was performed comparing the expression data from CD $19^{\text {hi }}$ and CD19lo tumors. The data from Lenz and Hummel studies were also used to evaluate the effect of CD19 and MYC expression on patient survival, as the 2 studies had similar design and the data could be combined. Using the pooled data, we calculated the tertiles of the normalized CD19 and MYC values and categorized patients into 3 groups: CD19 (or MYC) low, CD19 (or MYC) intermediate, and CD19 (or MYC) high. We compared the KM curves of the CD19 (or MYC) high group and CD19 (or MYC) low group.

Statistics. For the survival analysis, we conducted a stratified log-rank test, testing the effect of CD19 on survival and stratifying based on the study number (58) to account for the potential difference in the 2 studies. A 2-sided $P$ value of less than 0.05 was considered statistically significant, and SAS 9.2 was used to perform the analysis. For GSEA, a nominal $P$ value of less than 0.05 , in combination with an FDR $q$ value of less than 0.25 , was considered statistically significant.

Study approval. Animal studies described herein have been approved by The Children's Hospital of Philadelphia Institutional Animal Care and Use Committee (protocol no. 2009-12-902).

\section{Acknowledgments}

We are grateful to all members of PIG (Pathology Immunology Group at Penn), in particular Michael Cancro, David Allman, and Avinash Bhandoola for many stimulating discussions. We thank Michael Cole (Dartmouth Medical School) for his advise regarding the use of Thr-58 MYC mutants. We also thank Richard Aplenc for his advice regarding statistical analyses. This work was supported by NIH grants R01 CA102709 (to A. Thomas-Tikhonenko), T32 HL007439 (to E.Y. Chung), and T32 CA115299 (to J.N. Psathas) and grants from the V Foundation and WW Smith Charitable Trust (to A. Thomas-Tikhonenko). E.Y. Chung was a fellow of the Leukemia and Lymphoma Society (grant no. 5259-09).

Received for publication October 28, 2011, and accepted in revised form March 27, 2012.

Address correspondence to: Andrei Thomas-Tikhonenko, 4056 Colket Translational Research Building, 3501 Civic Center Blvd., Philadelphia, Pennsylvania 19104-4399, USA. Phone: 267.426.9699; Fax: 267.426.8125; E-mail: andreit@mail.med.upenn.edu.
1. Felsher DW. Cancer revoked: oncogenes as therapeutic targets. Nat Rev Cancer. 2003;3(5):375-380.

2. Rowley JD. Chromosome translocations: dangerous liaisons revisited. Nat Rev Cancer. 2001;1(3):245-250.

3. Druker BJ. Perspectives on the development of a molecularly targeted agent. Cancer Cell. 2002; $1(1): 31-36$

4. Taub R, et al. Translocation of the c-myc gene into the immunoglobulin heavy chain locus in human Burkitt lymphoma and murine plasmacytoma cells. Proc Natl Acad Sci U S A. 1982;79(24):7837-7841.

5. Dalla-Favera R, et al. Human c-myc onc gene is located on the region of chromosome 8 that is translocated in Burkitt lymphoma cells. Proc Natl
Acad Sci U S A. 1982;79(24):7824-7827.

6. Shen-Ong GL, Keath EJ, Piccoli SP, Cole MD. Novel myc oncogene RNA from abortive immunoglobulin-gene recombination in mouse plasmacytomas. Cell. 1982;31(2 pt 1):443-452.

7. Busslinger M. Transcriptional control of early B cell development. Annu Rev Immunol. 2004;22:55-79.

8. Monroe JG. ITAM-mediated tonic signalling through pre-BCR and BCR complexes. Nat Rev Immunol. 2006;6(4):283-294.

9. Maier H, et al. Requirements for selective recruitment of Ets proteins and activation of mb-1/Igalpha gene transcription by Pax-5 (BSAP). Nucleic Acids Res. 2003;31(19):5483-5489.
10. Schamel WW, Reth M. Monomeric and oligomeric complexes of the B cell antigen receptor. Immunity. 2000;13(1):5-14.

11. Nutt SL, Morrison AM, Dorfler P, Rolink A, Busslinger M. Identification of BSAP (Pax-5) target genes in early B-cell development by loss- and gain-of-function experiments. EMBOJ. 1998;17(8):2319-2333.

12. Kozmik Z, Wang S, Dorfler P, Adams B, Busslinger $\mathrm{M}$. The promoter of the CD19 gene is a target for the B-cell-specific transcription factor BSAP. $\mathrm{Mol}$ Cell Biol. 1992;12(6):2662-2672.

13. Offit K, Parsa NZ, Filippa D, Jhanwar SC, Chaganti RS. t(9;14)(p13;q32) denotes a subset of low-grade non-Hodgkin's lymphoma with plasmacytoid dif- 
ferentiation. Blood. 1992;80(10):2594-2599.

14. Cook JR, et al. Lack of PAX5 rearrangements in lymphoplasmacytic lymphomas: reassessing the reported association with $\mathrm{t}(9 ; 14)$. Hum Pathol. 2004;35(4):447-454.

15. Busslinger M, Klix N, Pfeffer P, Graninger PG, Kozmik Z. Deregulation of PAX-5 by translocation of the E $\mu$ enhancer of the IgH locus adjacent to two alternative PAX-5 promoters in a diffuse large-cell lymphoma. Proc Natl Acad Sci U S A. 1996; 93(12):6129-6134.

16. Iida $\mathrm{S}$, et al. The $\mathrm{t}(9 ; 14)(\mathrm{p} 13 ; \mathrm{q} 32)$ chromosomal translocation associated with lymphoplasmacytoid lymphoma involves the PAX-5 gene. Blood. 1996; 88(11):4110-4117.

17. Morrison AM, Jager U, Chott A, Schebesta M, Haas OA, Busslinger M. Deregulated PAX-5 transcription from a translocated $\mathrm{IgH}$ promoter in marginal zone lymphoma. Blood. 1998;92(10):3865-3878.

18. Poppe $\mathrm{B}$, et al. PAX5/IGH rearrangement is a recurrent finding in a subset of aggressive B-NHL with complex chromosomal rearrangements. Genes Chromosomes Cancer. 2005;44(2):218-223.

19. Pasqualucci L, et al. Hypermutation of multiple proto-oncogenes in B-cell diffuse large-cell lymphomas. Nature. 2001;412(6844):341-346.

20. Bousquet M, et al. A novel PAX5-ELN fusion protein identified in B-cell acute lymphoblastic leukemia acts as a dominant negative on wild-type PAX5. Blood. 2007;109(8):3417-3423.

21. Cazzaniga $\mathrm{G}$, et al. The paired box domain gene PAX5 is fused to ETV6/TEL in an acute lymphoblastic leukemia case. Cancer Res. 2001;61(12):4666-4670.

22. Mullighan CG, et al. Genome-wide analysis of genetic alterations in acute lymphoblastic leukaemia. Nature. 2007;446(7137):758-764.

23. Shaffer AL, Rosenwald A, Staudt LM. Lymphoid malignancies: the dark side of B-cell differentiation. Nat Rev Immunol. 2002;2(12):920-932.

24. Gururajan M, Jennings CD, Bondada S. Cutting edge: constitutive $B$ cell receptor signaling is critical for basal growth of B lymphoma. J Immunol. 2006; 176(10):5715-5719.

25. Staudt LM. It's ALL in the diagnosis. Cancer Cell. 2002;1(2):109-110.

26. Thomas-Tikhonenko A, Cozma D. PAX5 and B-cell neoplasms: transformation through presentation. Future Oncol. 2008;4(1):5-9.

27. Cozma D, et al. PAX5 promotes lymphomagenesis through the stimulation of B-cell receptor signaling. J Clin Invest. 2007;117(9):2602-2610.

28. Grande SM, Katz E, Crowley JE, Bernardini MS, Ross SR, Monroe JG. Cellular ITAM-containing proteins are oncoproteins in nonhematopoietic cells. Oncogene. 2006;25(19):2748-2757.

29. Refaeli Y, Young RM, Turner BC, Duda J, Field KA, Bishop JM. The B cell antigen receptor and overexpression of MYC can cooperate in the genesis of B cell lymphomas. PLoS Biol. 2008;6(6):e152.

30. Yu D, Allman D, Goldscmidt M, Atchison M, Monroe JG, Thomas-Tikhonenko A. Oscillation between B-lymphoid and myeloid lineages in Mycinduced hematopoietic tumors following spontaneous silencing/reactivation of the EBF/Pax5 pathway. Blood. 2003;101(5):1950-1955.

31. Johnson K, et al. B cell-specific loss of histone 3 lysine 9 methylation in the $\mathrm{V}(\mathrm{H})$ locus depends on Pax5. Nat Immunol. 2004;5(8):853-861.

32. Hodawadekar S, Wei F, Yu D, Thomas-Tikhonenko A, Atchison M. Epigenetic histone modifications do not control Igk locus contraction and intranuclear localization in cells with dual B cell-macrophage potential. J Immunol. 2006;177(9):6165-6171.

33. Hodawadekar S, Yu D, Freedman B, Sunyer JO, Atchison M, Thomas-Tikhonenko A. B-lymphoma cells with epigenetic silencing of Pax5 trans-differ- entiate into macrophages, but not other hematopoietic lineages. Exp Cell Res. 2007;313(2):331-340. 34. Pajic A, et al. Cell cycle activation by c-myc in a Burkitt lymphoma model cell line. Int J Cancer. 2000; 87(6):787-793.

35. Clark MR, et al. The B cell antigen receptor complex: association of Ig-alpha and Ig-beta with distinct cytoplasmic effectors. Science. 1992;258(5079):123-126.

36. Rickert RC, Rajewsky K, Roes J. Impairment of Tcell-dependent B-cell responses and B-1 cell development in CD19-deficient mice. Nature. 1995; 376(6538):352-355.

37. Schneider-Poetsch T, et al. Inhibition of eukaryotic translation elongation by cycloheximide and lactimidomycin. Nat Chem Biol. 2010;6(3):209-217.

38. Tuveson DA, Carter RH, Soltoff SP, Fearon DT. CD19 of B cells as a surrogate kinase insert region to bind phosphatidylinositol 3-kinase. Science. 1993; 260(5110):986-989

39. Buhl AM, Cambier JC. Phosphorylation of CD19 Y484 and Y515, and linked activation of phosphatidylinositol 3-kinase, are required for B cell antigen receptor-mediated activation of Bruton's tyrosine kinase. J Immunol. 1999;162(8):4438-4446.

40. Otero DC, Omori SA, Rickert RC. Cd19-dependent activation of Akt kinase in B-lymphocytes. J Biol Chem. 2001;276(2):1474-1478.

41. Fujimoto M, Poe JC, Satterthwaite AB, Wahl MI, Witte ON, Tedder TF. Complementary roles for CD19 and Bruton's tyrosine kinase in B lymphocyte signal transduction. J Immunol. 2002; 168(11):5465-5476

42. Aiba Y, Kameyama M, Yamazaki T, Tedder TF, Kurosaki T. Regulation of B-cell development by BCAP and CD19 through their binding to phosphoinositide 3-kinase. Blood. 2008;111(3):1497-1503.

43. Gold MR, et al. Targets of B-cell antigen receptor signaling: the phosphatidylinositol 3-kinase/Akt/ glycogen synthase kinase-3 signaling pathway and the Rap1 GTPase. Immunol Rev. 2000;176:47-68.

44. Hann SR. Role of post-translational modifications in regulating c-Myc proteolysis, transcriptional activity and biological function. Semin Cancer Biol. 2006;16(4):288-302.

45. Sears RC. The life cycle of c-myc: from synthesis to degradation. Cell Cycle. 2004;3(9):1133-1137.

46. Welcker M, et al. The Fbw7 tumor suppressor regulates glycogen synthase kinase 3 phosphorylationdependent c-Myc protein degradation. Proc Natl Acad Sci U S A. 2004;101(24):9085-9090.

47. Lutterbach B, Hann SR. Hierarchical phosphorylation at $\mathrm{N}$-terminal transformation-sensitive sites in c-Myc protein is regulated by mitogens and in mitosis. Mol Cell Biol. 1994;14(8):5510-5522.

48. Bello-Fernandez C, Packham G, Cleveland JL. The ornithine decarboxylase gene is a transcriptional target of c-Myc. Proc Natl Acad Sci U S A. 1993; 90(16):7804-7808

49. Schuhmacher $M$, et al. The transcriptional program of a human B cell line in response to Myc. Nucl Acids Res. 2001;29(2):397-406.

50. Elkon R, Zeller KI, Linhart C, Dang CV, Shamir R, Shiloh Y. In silico identification of transcriptional regulators associated with c-Myc. Nucl Acids Res. 2004;32(17):4955-4961.

51. Hermeking $\mathrm{H}$, et al. Identification of CDK4 as a target of c-MYC. Proc Natl Acad Sci U S A. 2000; 97(5):2229-2234.

52. Chang TC, et al. Widespread microRNA repression by Myc contributes to tumorigenesis. Nat Genet. 2008;40(1):43-50.

53. Hummel M, et al. A biologic definition of Burkitt's lymphoma from transcriptional and genomic profiling. N Engl J Med. 2006;354(23):2419-2430.

54. Hoang AT, et al. A link between increased transforming activity of lymphoma-derived myc mutant alleles, their defective regulation by p107, and altered phosphorylation of the c-myc transactivation domain. Mol Cell Biol. 1995; 15(8):4031-4042

55. Subramanian A, et al. Gene set enrichment analysis: a knowledge-based approach for interpreting genome-wide expression profiles. Proc Natl Acad Sci US A. 2005;102(43):15545-15550.

56. Zeller KI, Jegga AG, Aronow B, O’Donnell KA, Dang CV. An integrated database of genes responsive to the Myc oncogenic transcription factor: identification of direct genomic targets. Genome Biol. 2003;4(10):R69.

57. Lenz G, et al. Stromal gene signatures in large-B-cell lymphomas. NEngl J Med. 2008;359(22):2313-2323.

58. Kalbfleisch JD, Prentice R. The Statistical Analysis Of Failure Time Data. New York, New York, USA: John Wiley \& Sons, Inc.; 2002.

59. Tedder TF. CD19: a promising B cell target for rheumatoid arthritis. Nat Rev Rheumatol. 2009; 5(10):572-577.

60. Wang Y, Brooks SR, Li X, Anzelon AN, Rickert RC, Carter RH. The physiologic role of CD19 cytoplasmic tyrosines. Immunity. 2002;17(4):501-514.

61. Cross DA, Alessi DR, Cohen P, Andjelkovich M, Hemmings BA. Inhibition of glycogen synthase kinase- 3 by insulin mediated by protein kinase B. Nature. 1995;378(6559):785-789.

62. Pulverer BJ, Fisher C, Vousden K, Littlewood T, Evan GI, Woodgett JR. Site-specific modulation of c-Myc cotransformation by residues phosphorylated in vivo. Oncogene. 1994;9(1):59-70.

63. Srinivasan L, et al. PI3 kinase signals BCR-dependent mature B cell survival. Cell. 2009;139(3):573-586.

64. Patterson HC, Kraus M, Kim YM, Ploegh H, Rajewsky K. The B cell receptor promotes B cell activation and proliferation through a non-ITAM tyrosine in the Igalpha cytoplasmic domain. Immunity. 2006;25(1):55-65.

65. Kraus M, Alimzhanov MB, Rajewsky N, Rajewsky K. Survival of resting mature B lymphocytes depends on BCR signaling via the Ig $\alpha / \beta$ heterodimer. Cell. 2004;117(6):787-800.

66. Scheuermann RH, Racila E. CD19 antigen in leukemia and lymphoma diagnosis and immunotherapy. Leuk Lymphoma. 1995;18(5-6):385-397.

67. Davies JK, et al. Combining CD19 redirection and alloanergization to generate tumor-specific human $\mathrm{T}$ cells for allogeneic cell therapy of B-cell malignancies. Cancer Res. 2010;70(10):3915-3924.

68. Awan FT, et al. CD19 targeting of chronic lymphocytic leukemia with a novel Fc-domainengineered monoclonal antibody. Blood. 2010; 115(6):1204-1213.

69. Sapra P, Allen TM. Improved outcome when Bcell lymphoma is treated with combinations of immunoliposomal anticancer drugs targeted to both the CD19 and CD20 epitopes. Clin Cancer Res. 2004;10(7):2530-2537.

70. Chng WJ, et al. Clinical and biological implications of MYC activation: a common difference between MGUS and newly diagnosed multiple myeloma. Lenkemia. 2011;25(6):1026-1035.

71. Shou Y, et al. Diverse karyotypic abnormalities of the c-myc locus associated with c-myc dysregulation and tumor progression in multiple myeloma. Proc Natl Acad Sci U S A. 2000;97(1):228-233.

72. Delmore JE, et al. BET bromodomain inhibition as a therapeutic strategy to target c-Myc. Cell. 2011; 146(6):904-917.

73. Khwaja A. PI3K as a target for therapy in haematological malignancies. Curr Top Microbiol Immunol. 2010;347:169-188.

74. Chung EY, et al. c-Myb oncoprotein is an essential target of the dleu2 tumor suppressor microRNA cluster. Cancer Biol Ther. 2008;7(11):1758-1764. 